\title{
Tetrahydrobiopterin analogues with NO-dependent pulmonary vasodilator properties
}

\author{
Suma P. Kunuthur ${ }^{\mathrm{a}, 1}$, Philip H. Milliken ${ }^{\mathrm{a}, 2}$, Colin L. Gibson ${ }^{\mathrm{b}}$, Colin J. Suckling ${ }^{\mathrm{b}}$, Roger M. Wadsworth ${ }^{\mathrm{a}, *}$ \\ a Strathclyde Institute of Pharmacy and Biomedical Sciences, UK \\ ${ }^{\mathrm{b}}$ Department of Pure and Applied Chemistry, University of Strathclyde, Glasgow, UK
}

\section{A R T I C L E I N F O}

Article history:

Received 10 May 2010

Received in revised form 2 September 2010

Accepted 23 September 2010

Available online 13 October 2010

\section{Keywords:}

Tetrahydrobiopterin

Pulmonary artery

Nitric oxide

Pulmonary hypertension

Nitric oxide synthase

\begin{abstract}
A B S T R A C T
Reduced NO levels due to the deficiency of tetrahydrobiopterin $\left(\mathrm{BH}_{4}\right)$ contribute to impaired vasodilation in pulmonary hypertension. Due to the chemically unstable nature of $\mathrm{BH}_{4}$, it was hypothesised that oxidatively stable analogues of $\mathrm{BH}_{4}$ would be able to support $\mathrm{NO}$ synthesis to improve endothelial dysfunction in pulmonary hypertension. Two analogues of $\mathrm{BH}_{4}$, namely 6-hydroxymethyl pterin (HMP) and 6-acetyl-7,7dimethyl-7,8-dihydropterin (ADDP), were evaluated for vasodilator activity on precontracted rat pulmonary artery rings. ADDP was administered to pulmonary hypertensive rats, followed by measurement of pulmonary vascular resistance in perfused lungs and eNOS expression by immunohistochemistry. ADDP and HMP caused significant relaxation in vitro in rat pulmonary arteries depleted of $\mathrm{BH}_{4}$ with a maximum relaxation at $0.3 \mu \mathrm{M}$ (both $\mathrm{P}<0.05$ ). Vasodilator activity of ADDP and HMP was completely abolished following preincubation with the NO synthase inhibitor, L-NAME. ADDP and HMP did not alter relaxation induced by carbachol or spermine NONOate. $\mathrm{BH}_{4}$ itself did not produce relaxation. In rats receiving ADDP $14.1 \mathrm{mg} / \mathrm{kg} / \mathrm{day}$, pulmonary vasodilation induced by calcium ionophore A23187 was augmented and eNOS immunoreactivity was increased. In conclusion, ADDP and HMP are two analogues of $\mathrm{BH}_{4}$, which can act as oxidatively stable alternatives to $\mathrm{BH}_{4}$ in causing NO-mediated vasorelaxation. Chronic treatment with ADDP resulted in improvement of NO-mediated pulmonary artery dilation and enhanced expression of eNOS in the pulmonary vascular endothelium. Chemically stable analogues of $\mathrm{BH}_{4}$ may be able to limit endothelial dysfunction in the pulmonary vasculature.
\end{abstract}

(C) 2010 Elsevier B.V. All rights reserved.

\section{Introduction}

Deficiency in NO formation has been found in patients with primary pulmonary hypertension (Demoncheaux et al., 2005) and in the hypoxic rat model of pulmonary hypertension (Weerackody et al., 2009). Tetrahydrobiopterin $\left(\mathrm{BH}_{4}\right)$ is an essential cofactor for nitric oxide synthase (NOS), and mice that have very low $\mathrm{BH}_{4}$ tissue levels exhibit pulmonary hypertension (Khoo et al., 2005), which could be reversed by increasing $\mathrm{BH}_{4}$ through targeted transgenic overexpression of GTP cyclohydrolase 1 (GTPCH-1) (Khoo et al., 2005). Using an experimental animal model of persistent pulmonary hypertension of the newborn (PPHN), it was found that the oxidised form, dihydrobiopterin $\left(\mathrm{BH}_{2}\right)$ is raised in the lungs (Grobe et al., 2006). In

\footnotetext{
* Corresponding author. Strathclyde Institute of Pharmacy and Biomedical Sciences, University of Strathclyde, Glasgow G 4 0NR, UK. Tel.: +440141548 2154.

E-mail address: r.m.wadsworth@strath.ac.uk (R.M. Wadsworth).

1 Current address: The Hatter Cardiovascular Institute, University College London, 67 Chenies Mews, London WC1E 6HX.

${ }^{2}$ Current address: GlaxoSmithKline, Safety Pharmacology, Park Road, Ware, Hertfordshire, SG12 ODP.
}

another animal model of PPHN, it was found that the levels of GTPCH1 were depressed; moreover the administration of $\mathrm{BH}_{4}$ combined with the superoxide dismutase mimetic MnTMPyP improved pulmonary artery relaxation (Nandi et al., 2006).

$\mathrm{BH}_{4}$ depletion is believed to be due to oxidative stress since SOD overexpression restores both $\mathrm{BH}_{4}$ content and activity of GTPCH-1, the rate-limiting enzyme in $\mathrm{BH}_{4}$ synthesis (Farrow et al., 2008). The endothelial NO synthase (eNOS) enzyme generates superoxide when $\mathrm{BH}_{4}$ levels decline or when it is replaced by an oxidised product, $\mathrm{BH}_{2}$ (Crabtree et al., 2009; Vasquez-Vivar et al., 2002).

In vitro administration of $\mathrm{BH}_{4}$, or its physiological precursor sepiapterin, restored endothelium-dependent relaxation of resistance arteries in animal models of cardiovascular disease (Pannirselvam et al., 2003; Tiefenbacher et al., 2000) and in patients with endothelium dysfunction (Heitzer et al., 2000a,b; Higashi et al., 2006; Setoguchi et al., 2002). Since $\mathrm{BH}_{4}$ is rapidly oxidised under physiological conditions, oral administration requires very high doses and is only briefly effective. Oral administration of $\mathrm{BH}_{4}$ has been reported to improve endothelium function (Cosentino et al., 2008; Eskurza et al., 2005) and to reduce blood markers of oxidative stress in patients (Cosentino et al., 2008). Administration of $\mathrm{BH}_{4}$ attenuated the rise in pulmonary artery pressure and reduced muscularisation of 
distal pulmonary arteries in rats with monocrotaline-induced pulmonary hypertension (Francis et al., 2009).

We believe that analogues of $\mathrm{BH}_{4}$ with stable oxidative states might be better alternatives to improve impaired relaxation associated with endothelial dysfunction and will indirectly substitute for $\mathrm{BH}_{4}$ in the vasculature. To explore this possibility, two analogues of $\mathrm{BH}_{4}$, namely 6-hydroxymethyl pterin (HMP) and 6-acetyl-7,7dimethyl-7,8-dihydropterin (ADDP), were obtained from the products of a large synthetic programme to generate biologically active pteridines (Al Hassan et al., 1985).They were selected for their chemical stability and similarity to the structure of $\mathrm{BH}_{4}$ (Suckling et al., 2008). The tetrahydro derivative of HMP has been shown to be a $\mathrm{BH}_{4}$ substitute (Kotsonis et al., 2001).

In the current study, we investigated the effects of these two analogues on normal as well as $\mathrm{BH}_{4}$-depleted pulmonary arteries. We also report in vivo administration of ADDP on endotheliumdependent vasorelaxation in a rat model of hypoxic pulmonary hypertension.

\section{Methods}

\subsection{Artery ring experiments}

Male Sprague-Dawley rats of body weight approximately 175$350 \mathrm{~g}$ were killed by cervical dislocation. The heart and lungs were placed in either the chilled Krebs-Henseleit solution in experiments where normal untreated arteries were used and in chilled tissue culture medium when the artery rings were treated to deplete the endogenous levels of $\mathrm{BH}_{4}$. The main pulmonary artery along with its right and left extra pulmonary branches were dissected and divided into four rings each $2-3 \mathrm{~mm}$ long. Pulmonary artery rings were mounted on to a pair of intraluminal wires under $1 \mathrm{~g}$ resting force in $15 \mathrm{ml}$ organ baths containing oxygenated $\left(20 \% \mathrm{O}_{2}, 5 \% \mathrm{CO}_{2}\right.$ and $75 \% \mathrm{~N}_{2}$ gas mixture) Krebs-Henseleit solution at $37^{\circ} \mathrm{C}$ and contractile force was measured.

Depletion of cellular $\mathrm{BH}_{4}$ was carried out using 2,4-diamino-6hydroxypyrimidine (DAHP), which inhibits GTPCH1, the key ratelimiting enzyme in the synthesis of endogenous $\mathrm{BH}_{4}$ (Bagi and Koller, 2003; Kinoshita et al., 1997; Wang et al., 2008). Artery rings were incubated for $6 \mathrm{~h}$ at $37^{\circ} \mathrm{C}$ in minimum essential medium containing DAHP $\left(10^{-2} \mathrm{M}\right)$ (Kinoshita et al., 1997). Control arteries received similar incubation omitting DAHP.

In experiments showing the effects of $\mathrm{BH}_{4}, \mathrm{ADDP}$ or $\mathrm{HMP}$ in normal or $\mathrm{BH}_{4}$-depleted arteries the following protocol was used. After $1 \mathrm{~h}$ equilibration of the tissue, arteries were precontracted with phenylephrine: $3.6 \times 10^{-8} \mathrm{M}$ (the $\mathrm{EC}_{50}$ concentrations determined from preliminary studies) followed by relaxation with $10^{-5} \mathrm{M}$ carbachol to check for the integrity of the endothelium. Preparations were rejected if they did not produce a minimum of $80 \%$ relaxation to carbachol. After washout tissues were again contracted with phenylephrine followed by the test drugs ( $\mathrm{BH}_{4}, \mathrm{ADDP}$ or HMP). One parallel ring was used as a time control and another was a vehicle control (receiving the relevant concentrations of $\mathrm{NH}_{4} \mathrm{OH}$ present in the parallel ring treated with ADDP or HMP). Relaxation by the drugs was measured as reduction in the percentage of precontraction obtained by phenylephrine.

To investigate if ADDP or HMP induce relaxation through NO, the tissues after the initial contraction and relaxation with phenylephrine and carbachol respectively were incubated with $N^{\mathrm{G}}$-nitro- ${ }_{\mathrm{L}}$-arginine methyl ester (L-NAME, $300 \mu \mathrm{M}$ ) for $30 \mathrm{~min}$ and then the effects of the analogues were studied.

In order to investigate if ADDP or HMP elicits a protective function on the already existing NO in the tissues, the rings were contracted with phenylephrine and a dose-response curve to NO donor, spermine NONOate, was produced followed by washing and equilibration for $30 \mathrm{~min}$ and then the rings were preconstricted with phenylephrine and incubated with a single concentration of the analogues $\left(3 \times 10^{-7} \mathrm{M}\right)$ at which maximum relaxation was originally seen followed by another dose-response curve to spermine NONOate.

In order to investigate the hypothesis that $\mathrm{O}_{2}^{--}$production might be leading to the contraction generated by $\mathrm{BH}_{4}$, we studied the effects of superoxide scavenger, $\mathrm{Mn}(\mathrm{III})$ tetrakis [1-methyl-4-pyridyl] porphyrin (MnTMPyP), on the $\mathrm{BH}_{4}$-induced contraction. Arteries were preconstricted with phenylephrine and a concentration-response curve to $\mathrm{BH}_{4}$ was generated. All drugs were then washed out and MnTMPyP $(30 \mu \mathrm{M})$ was incubated for $30 \mathrm{~min}$. Previous studies performed in the lab have shown that the above incubation parameters are optimal for scavenging $\mathrm{O}_{2}^{--}$by MnTMPyP. Following incubation with MnTMPyP and preconstriction with phenylephrine, a final concentration response curve to $\mathrm{BH}_{4}$ was produced in the presence of MnTMPyP.

The values are expressed as means \pm standard errors of means (S.E.M.) where $\mathrm{n}=$ number of arterial rings from separate animals. Statistical tests were performed using analysis of variance general linear model (repeated measures design). Repeated measures design was used to compare two cumulative dose response curves generated in two different tissues. A crossover design was used to compare two cumulative curves generated before and after they were treated with the test drug. The statistical package used was Minitab. The differences were treated significant if the P value $\leq$ 0.05 .

\subsection{Chronic hypoxic rats}

All procedures under this project have been performed under the UK Animals (Scientific Procedures) Act 1986. Male Sprague-Dawley rats of body weight $125-150 \mathrm{~g}$ were placed in a hypobaric chamber at 600 millibar pressure for a 14 day period. Animals in the hypobaric chamber had pulmonary hypertension (mean pulmonary arterial pressures in hypoxic rats $20.1 \pm 0.9 \mathrm{~mm} \mathrm{Hg}$; in normoxic rats $11.9 \pm$ $0.9 \mathrm{~mm} \mathrm{Hg} ; \mathrm{P} \leq 0.0001 ; \mathrm{n}=8-10)$ and right ventricular hypertrophy (ratio of right ventricular weight/weight of the left ventricle + septum in the hypoxic rats $0.41 \pm 0.007$ compared to the normoxic control rats $0.26 \pm 0.004, \mathrm{P} \leq 0.0001 ; \mathrm{n}=31)$. At the end of this period the rats were deeply anaesthetized with sodium pentobarbitone $(2 \mathrm{ml} / \mathrm{kg}$ intraperitoneal injection) and ventilated. The thorax was opened and the pulmonary artery was cannulated and perfused with heparinized Krebs-Henseleit solution ( $119 \mathrm{mM} \mathrm{NaCl}, 25 \mathrm{mM} \mathrm{NaHCO}, 11 \mathrm{mM}$ glucose, $4.6 \mathrm{mM} \mathrm{KCl}, 1.2 \mathrm{mM} \mathrm{MgCl}, 1.2 \mathrm{mM} \mathrm{KH}_{2} \mathrm{PO}_{4}$ and $2.5 \mathrm{mM}$ $\mathrm{CaCl}_{2}$ with 100 units/ml heparin, $4 \%$ albumin and $1 \mathrm{mM}$ flurbiprofen) in a recirculating circuit. Perfusion was constant flow with measurement of perfusion pressure at the pulmonary artery cannula. A cannula was inserted into the trachea for ventilation of the lungs with either a normoxic $\left(20 \% \mathrm{O}_{2}, 5 \% \mathrm{CO}_{2}\right.$, and $\left.75 \% \mathrm{~N}_{2}\right)$ or hypoxic $(0 \%$ $\mathrm{O}_{2}, 5 \% \mathrm{CO}_{2}$, and $95 \% \mathrm{~N}_{2}$ ) atmosphere.

Slight tone was induced in the perfused lungs using the thromboxane $A_{2}$ mimetic, 9,11-dideoxy-11 $\alpha, 9 \alpha$-epoxymethanoprostaglandin $F_{2 \alpha}\left(U 46619,3 \times 10^{-5} \mathrm{M}\right)$ given as a bolus dose to the perfusion circuit. Relaxation was induced using the endothelium-dependent vasorelaxant, calcium ionophore A23197, added in increasing doses. During the course of the experiments no visible lung oedema was observed and this was confirmed by measuring the weights of the lungs at the end of every experiment.

Drug-treated rats received ADDP during the period that they were in the hypoxic chamber. ADDP was administered to the rats via subcutaneous injections to give a dose of $14.1 \mathrm{mg} / \mathrm{kg} / \mathrm{day}$ as daily injections in the morning for both the hypertensive and the normotensive groups of rats. The ADDP solution for injection contained ADDP $100 \mathrm{mM}$ ADDP dissolved in dimethyl sulfoxide. The subcutaneous site of injection was in the neck region or on the back of the rats avoiding the spine, and was changed every day in order to prevent soreness at the site of injection. 
Immunohistochemistry was used to perform eNOS staining on the lung sections as previously described (Grant et al., 2006). Lungs from normoxic and chronically hypoxic rats, which have been treated with or without ADDP were perfused with formalin, dehydrated, and embedded in paraffin wax and $3 \mu \mathrm{m}$ sections were cut. The lung sections were rehydrated, treated with $0.3 \% \mathrm{H}_{2} \mathrm{O}_{2}$ for 10 min to block endogenous peroxidase activity and then heated by microwave to expose the antigens. The sections were blocked with $20 \%$ normal goat serum then incubated for $1 \mathrm{~h}$ with mouse monoclonal anti-human eNOS primary antibody (1:2000) (BD Transduction Laboratories). Sections were then treated with biotinylated secondary antibody (30 $\mathrm{min}$ ) followed by avidin-labelled horseradish-peroxidase (40 min) and then 0.5\% diaminobenzidine tetrahydrochloride activated with $30 \% \mathrm{H}_{2} \mathrm{O}_{2}(10 \mathrm{~min})$. The sections were counterstained with haematoxylin. The slides were coded so that the investigator was not aware of their identity during scoring. Sections were viewed under $400 \times$ magnification allocating intensity scores from 0-3 for each section, where 0 represents no staining, 1 is mild or uneven staining, 2 is moderate staining and 3 represents maximum staining observed with the antibody at the highest level of eNOS expression encountered. Intermediate intensity levels gave scores of $0.5,1.5$ and 2.5. In each staining run negative controls were included, where sections were not treated with primary antibody.

The values are expressed as means \pm standard errors of means (S.E.M.) where $\mathrm{n}=$ number of animals. Statistical tests were performed using analysis of variance general linear model (repeated measures design) to compare two cumulative dose-response curves generated in two different tissues, using Minitab. Histological scores were compared using Student's unpaired t-test. The differences were treated significant if the P value $\leq 0.05$.

\subsection{Drugs and solutions}

$\mathrm{BH}_{4}$ stock solution was stored at $-20{ }^{\circ} \mathrm{C}$ and minutes before the addition into the bath a single aliquot was taken out, thawed and diluted. Working solutions were kept on ice at all times when outside and protected from light. Stock solutions of the ADDP and HMP ( $2 \mathrm{mM}$ ) were dissolved in $2 \mathrm{M} \mathrm{NH}_{4} \mathrm{OH}$. Further dilutions were made with distilled water and these were stored at $4{ }^{\circ} \mathrm{C}$.
$\mathrm{BH}_{4}$, DAHP and L-NAME were bought from Sigma Aldrich Ltd, Poole, Dorset, UK; Spermine NONOate from Merck Biosciences Ltd, Beeston, Nottingham, UK; Ham's medium (Nutrient mixture HAM F12) and Waymouth medium MB 752/1 with L-glutamine were from Invitrogen Ltd, Paisley, UK. ADDP and HMP were synthesised as previously described (Suckling et al., 2008).

\section{Results}

\subsection{Artery ring experiments}

Both analogues of tetrahydrobiopterin produced dose-dependent relaxation in pulmonary arteries treated with DAHP. ADDP (6-acetyl7,7-dimethyl-7,8-dihydropterin ) produced a maximum relaxation at $0.3 \mu \mathrm{M}$ of $28.1 \pm 4.3 \%$ and HMP (6-hydroxymethyl pterin) produced a maximal relaxation at $0.3 \mu \mathrm{M}$ of $18.8 \pm 10.4 \%$ (Fig. 1A, B). ADDPinduced and HMP-induced relaxation were completely abolished following preincubation with the NO synthase inhibitor, L-NAME $(300 \mu \mathrm{M})$ (Fig. 1A, B). However, in pulmonary artery rings that had not been treated with DAHP, ADDP had no significant effect, and the relaxation generated by HMP was reduced (Fig. 1C, D).

Relaxation induced by the NO donor spermine NONOate was unaltered by co-administration of either ADDP $0.3 \mu \mathrm{M}$ or HMP $0.3 \mu \mathrm{M}$ in DAHP-pretreated artery rings (Fig. 2A, B). Similarly, incubation with either ADDP or HMP $(0.3 \mu \mathrm{M})$ did not have any effect on endothelium-dependent vasorelaxation caused by carbachol in pulmonary arteries pretreated with DAHP (Fig. 2C, D).

Contrary to the effects of the $\mathrm{BH}_{4}$ analogues ADDP and HMP, $\mathrm{BH}_{4}$ itself did not produce any significant relaxation over a concentration range of $10^{-8}-10^{-5} \mathrm{M}$. No relaxation occurred in pulmonary arteries, whether they had been pretreated with DAHP or not (Fig. 3A, B). Indeed the highest concentration of $\mathrm{BH}_{4}$ induced a significant contraction (Fig. 3A, B). In addition, in pulmonary arteries we found that incubation with $\mathrm{BH}_{4}(100 \mu \mathrm{M})$ resulted in reduction of potency of carbachol-induced relaxation at the initial concentrations without altering the maximum relaxation obtained (Fig. 4A). The superoxide scavenger, MnTMPyP $30 \mu \mathrm{M}$ did not prevent the contraction produced by $\mathrm{BH}_{4}$ in pulmonary arteries (Fig. 4B).
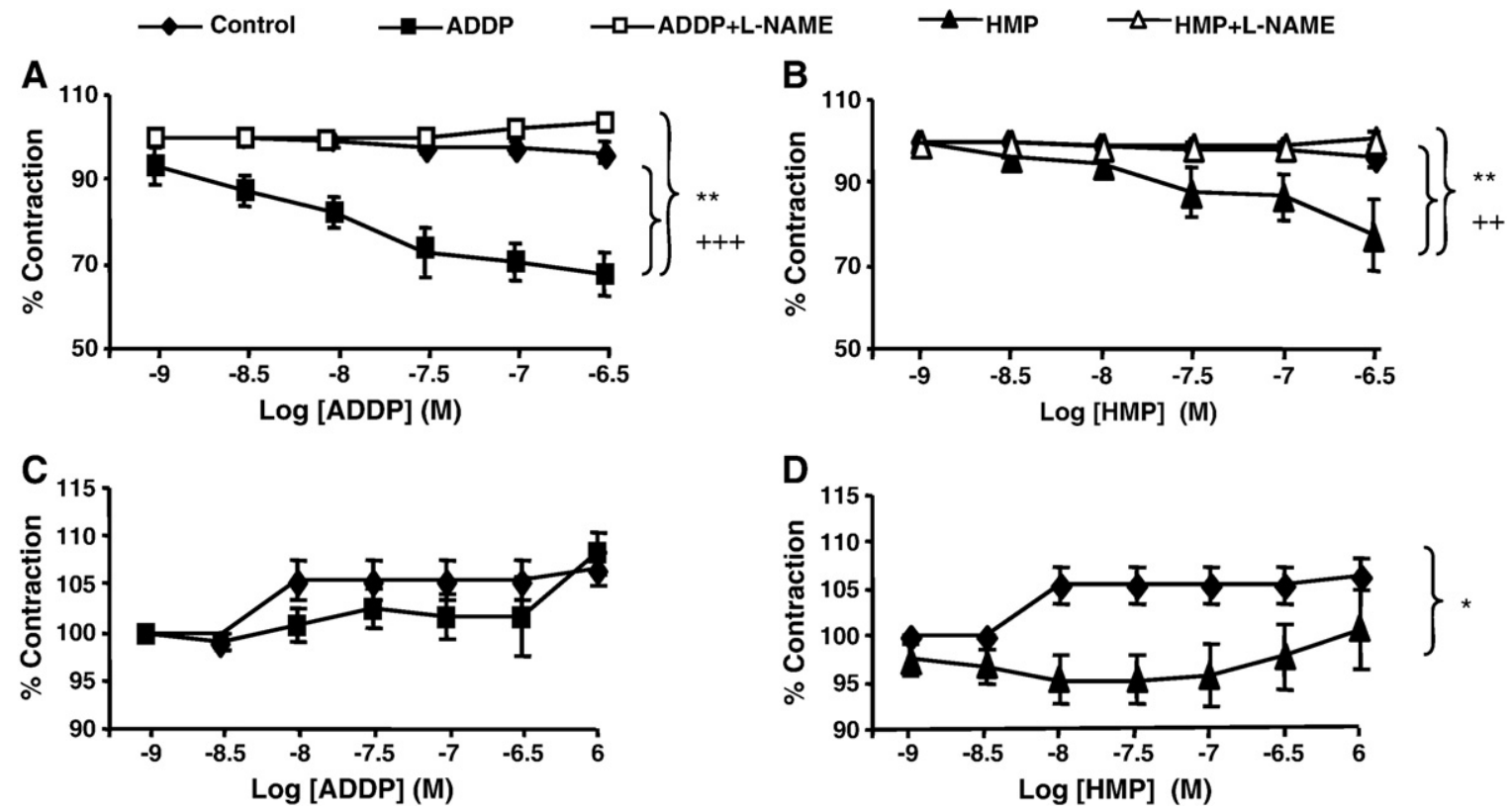

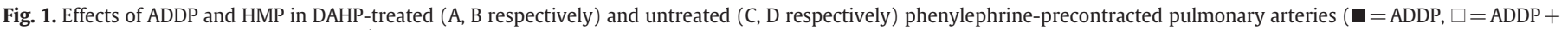

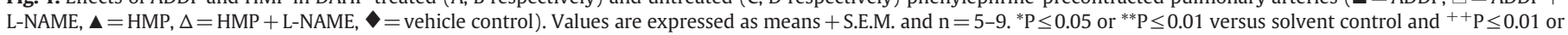
$++{ }^{+} \mathrm{P} \leq 0.001$ versus $\mathrm{L}-\mathrm{NAME}$. 

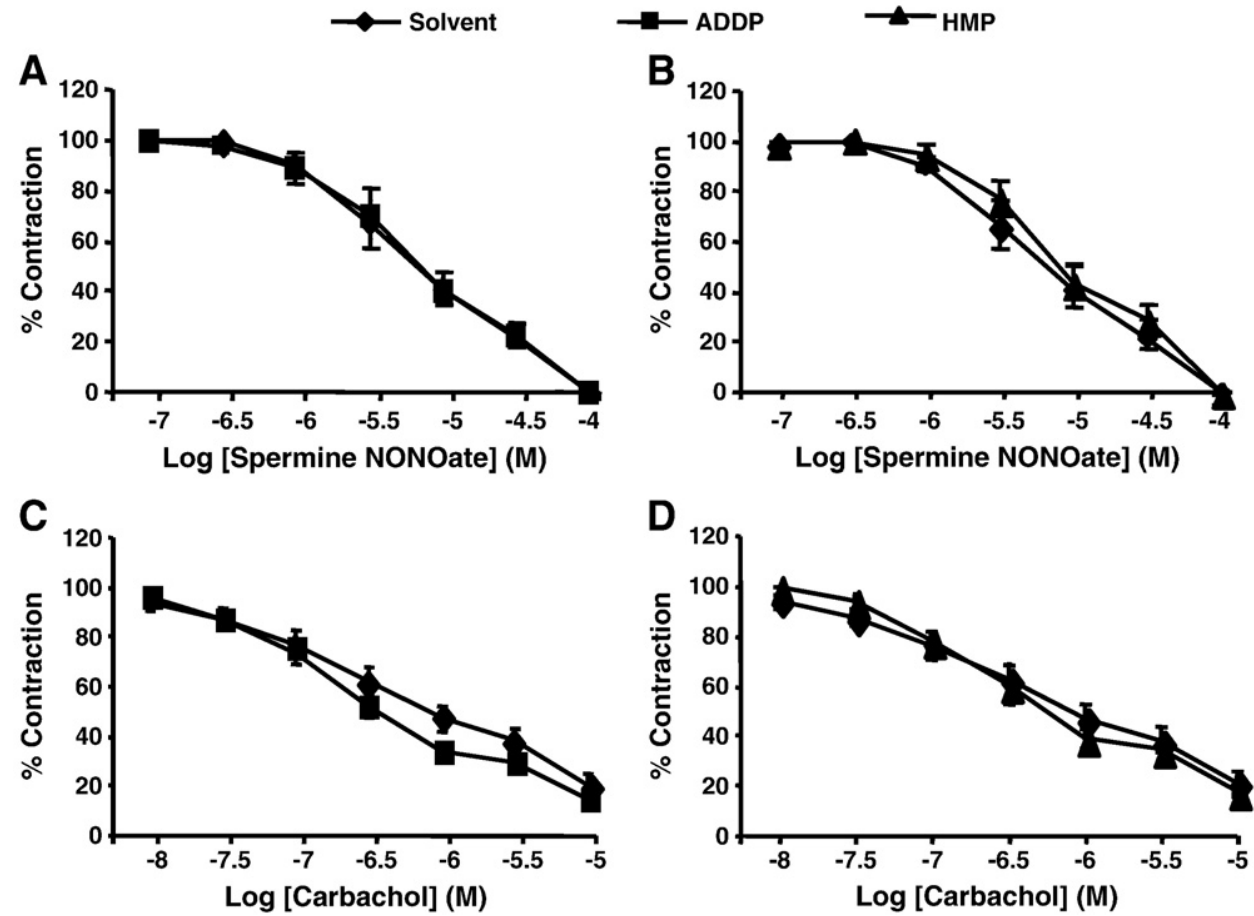

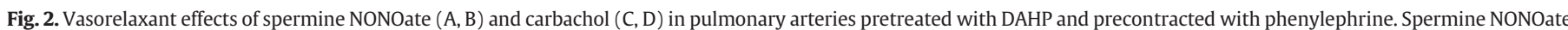
or carbachol was added in the presence of ADDP $0.3 \mu \mathrm{M}(\boldsymbol{\nabla})$ or HMP $0.3 \mu \mathrm{M}(\boldsymbol{\Delta})$ or vehicle $(\bullet)$. Values are expressed as means + S.E.M. and $\mathrm{n}=4-6$.

3.2. Effects of treatment with $A D D P$ on relaxation responses of calcium ionophore in isolated perfused lungs

Following preconstriction with U46619, responses to the endothelium-dependent vasorelaxant, calcium ionophore A23187, were measured in perfused lungs from pulmonary hypertensive and normotensive rats. Calcium ionophore A23187 completely relaxed the pulmonary circulation of both pulmonary hypertensive and normotensive rats. Chronic treatment of the rats with ADDP potentiated
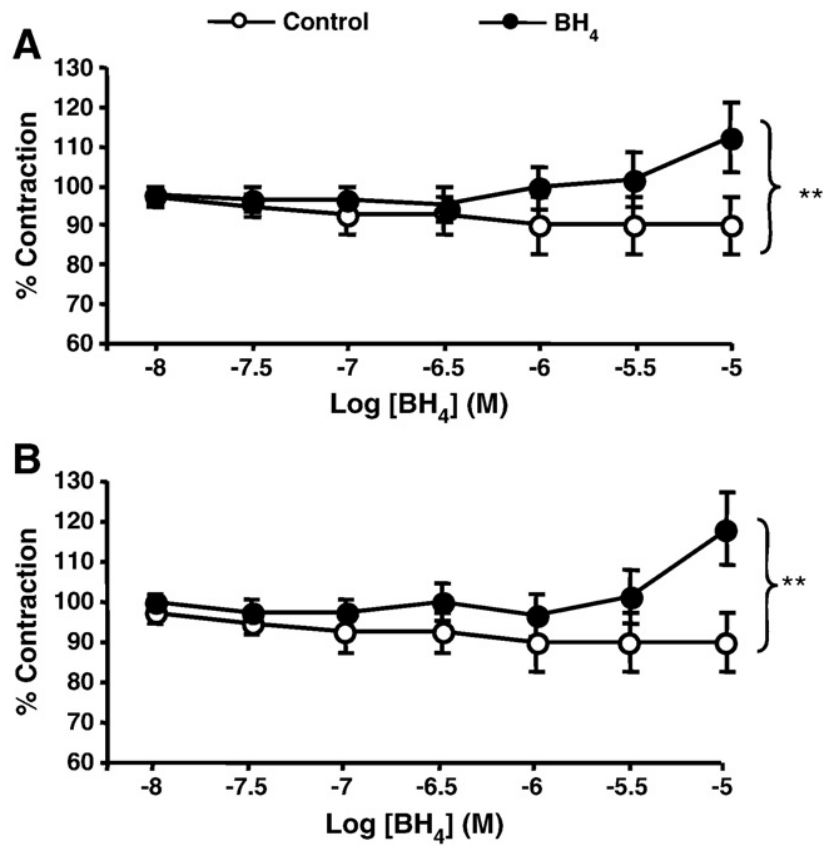

Fig. 3. Effects of $\mathrm{BH}_{4}$ in DAHP-pretreated (A) and untreated (B) and phenylephrineprecontracted pulmonary arteries $\left(\bullet=\mathrm{BH}_{4}\right.$ and $\mathrm{O}=$ time control. Values are expressed as means + S.E.M. and $n=7-11 .{ }^{* *} \mathrm{P} \leq 0.01$ versus time control. pulmonary relaxation in response to A23187 both in pulmonary hypertensive and in normotensive rats (Fig. 5A, B). Calculation of the $\mathrm{EC}_{50}$ values confirmed the potentiation effect of ADDP treatment in
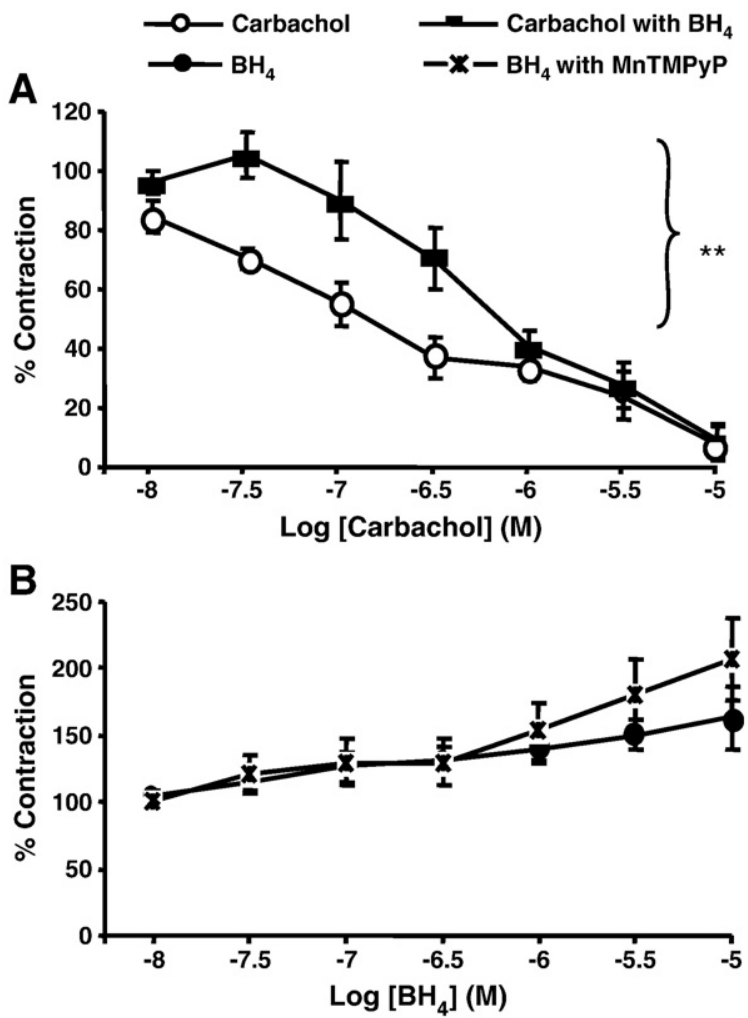

Fig. 4. (A) Vasorelaxant effects of carbachol in the presence or absence of $\mathrm{BH}_{4}$ in phenylephrine-precontracted pulmonary arteries $(\mathrm{O}=$ carbachol alone, $-=$ carbachol + $\mathrm{BH}_{4}$ ). (B) Effects of $\mathrm{BH}_{4}$ on phenylephrine-precontracted pulmonary arteries in the presence of MnTmPyP $30 \mu \mathrm{M}$. $\bullet=\mathrm{BH}_{4}, \mathbf{x}=\mathrm{BH}_{4}+$ MnTMPyP. Values are expressed as means + S.E.M. and $n=6$. ${ }^{* *} \mathrm{P} \leq 0.01$ carbachol versus carbachol $+\mathrm{BH}_{4}$ 

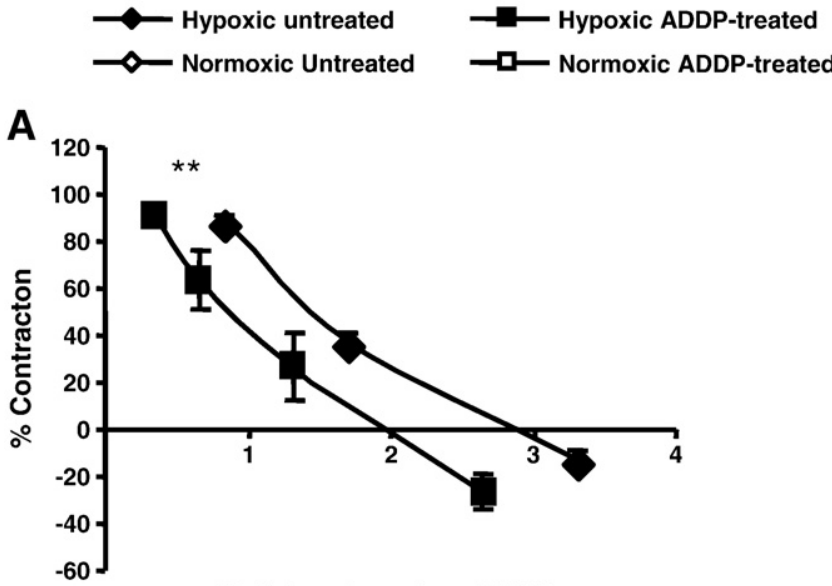

[Calcium lonophore] $(\mu \mathrm{M})$

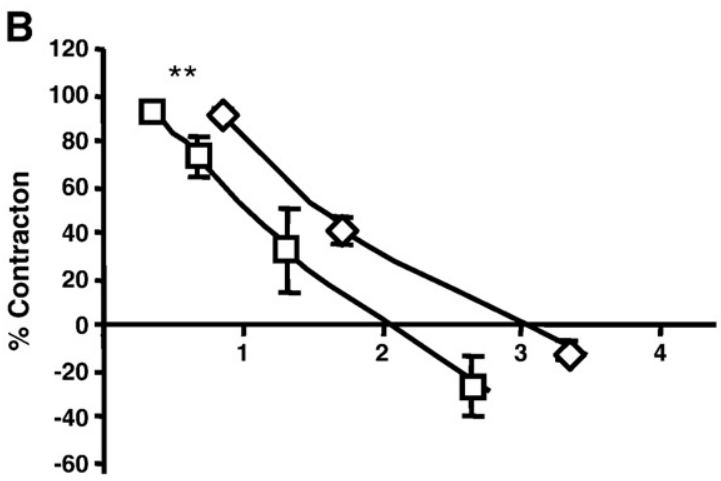

[Calcium lonophore] $(\mu \mathrm{M})$

Fig. 5. Vasorelaxant responses of calcium ionophore A23187 in U46619-precontracted (A) chronic hypoxic pulmonary hypertensive and (B) normotensive control rat lungs. (A) Chronic hypoxic rats were ADDP-treated ( $\mathbf{\square})$ or untreated ( $)$ ). (B) Matched control normotensive rats were ADDP-treated $(\square)$ or untreated $(\diamond)$. Rats were treated with ADDP $14.1 \mathrm{mg} / \mathrm{kg} /$ day s.c. $(\square, \boldsymbol{\square})$. Values are expressed as means \pm S.E.M. and $\mathrm{n}=8-20$. **P value $\leq 0.01$ comparing ADDP-treated with respective untreated groups.

both normoxic as well as hypoxic rat lungs as shown by the significant left-ward shift in the calcium ionophore A23187 dose-response curves generated in the ADDP-treated rat lungs compared to the untreated rat lungs $\left(1.1 \pm 0.1 \times 10^{-6} \mathrm{M}\right.$ versus $1.5 \pm 0.1 \times 10^{-6} \mathrm{M}$ in normoxic ADDP-treated and untreated rats respectively, $\mathrm{P}$ value $\leq$ $0.05, \mathrm{n}=8-20 ; 1.2 \pm 0.2 \times 10^{-6} \mathrm{M}$ versus $1.7 \pm 0.1 \times 10^{-6} \mathrm{M}$ in hypoxic ADDP-treated and untreated rats respectively, $\mathrm{P}$ value $\leq 0.05$, $\mathrm{n}=8-20)$.

\subsection{Effects of treatment with ADDP on eNOS expression}

Lung samples from untreated and ADDP-treated rats were stained for eNOS immunoreactivity. eNOS immunoreactivity was present throughout the vascular endothelium of small and large pulmonary arteries. Quantitative staining intensity analysis showed that eNOS immunostaining was significantly higher in the pulmonary hypertensive rat lungs when compared to the normotensive controls. Normotensive rats that had been treated with ADDP had significantly higher eNOS expression when compared to the normotensive rats that had received vehicle. In pulmonary hypertensive rats, eNOS expression was already at a high level and was not further increased by treatment with ADDP (Fig. 6).

\section{Discussion}

In the search for improved molecules that can reverse $\mathrm{BH}_{4}$ deficiency, sepiapterin has been administered to diabetic rats

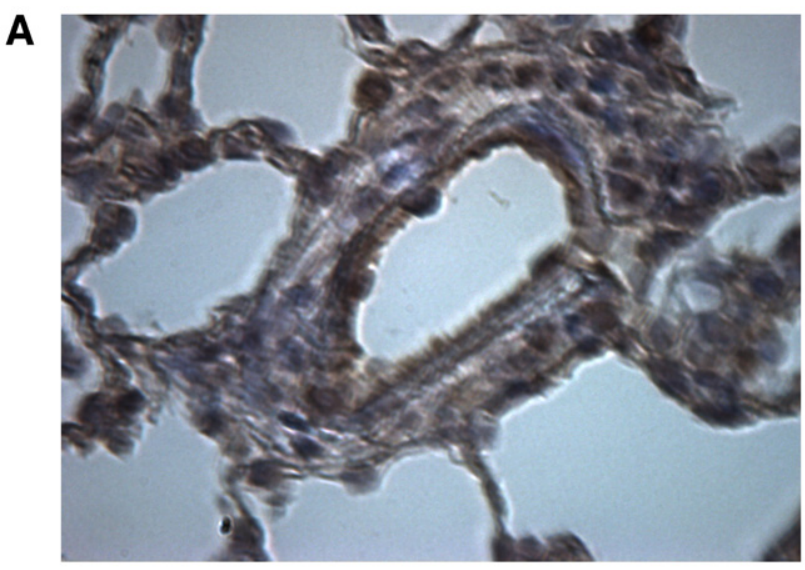

B

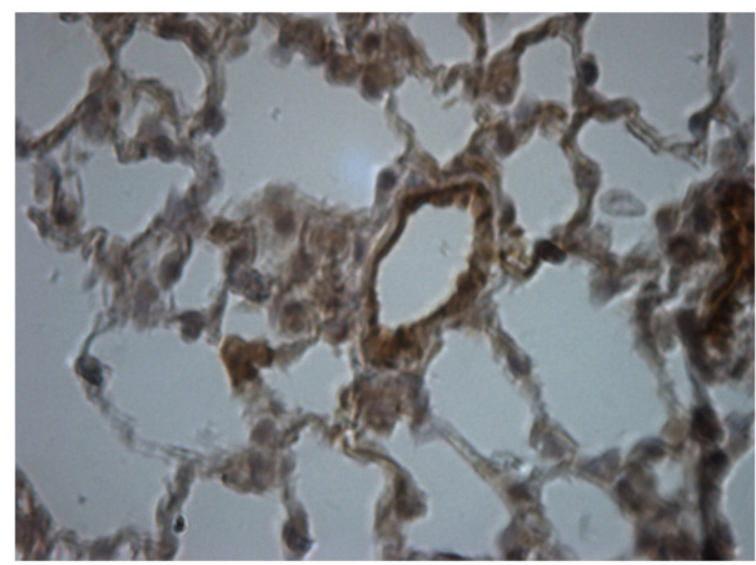

C

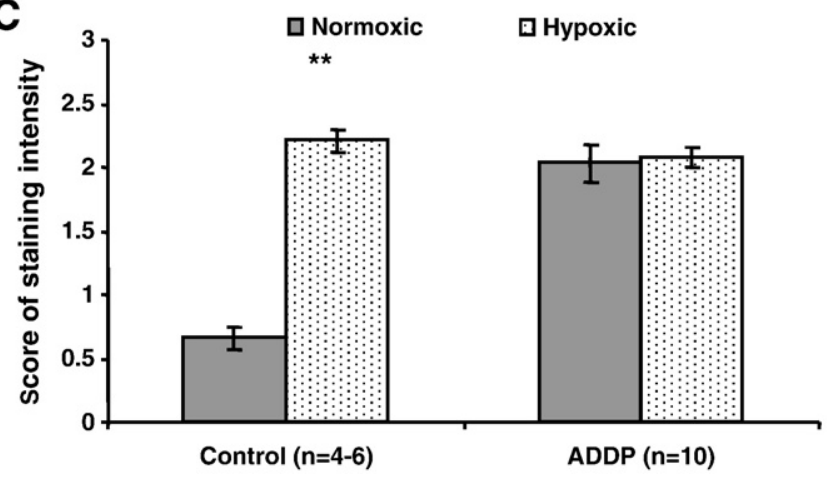

Fig. 6. Micrographs of small pulmonary arteries from lungs of rats treated with ADDP with immunohistochemistry for eNOS. (A) Pulmonary hypertensive rat, $\times 40$ objective (B) normotensive rat, $\times 40$ objective. (C) Quantitative measurement of eNOS staining in endothelial cells of small pulmonary arteries in control and ADDP-treated normoxic and chronic hypoxic rats. Values are expressed as means \pm S.E.M. and $n=4-10$. ${ }^{* *} \mathrm{P}$ value $\leq 0.01$ chronic hypoxic versus normoxic.

(Pannirselvam et al., 2003), however it requires conversion by sepiapterin reductase within cells. 5-Methylfolate has been found to improve endothelium function in rats that were depleted of $\mathrm{BH}_{4}$ (Hyndman et al., 2002), however it is not clear whether this relatively bulky molecule can bind stably to the pterin binding site on eNOS. Numerous analogues of $\mathrm{BH}_{4}$ have been synthesised, however where these have been tested they inhibit, not stimulate, eNOS (Bömmel et al., 1998; Fitzal et al., 2002; Fröhlich et al., 1999; Gibraeil et al., 2000; Gorren et al., 2000).

Our results show that the $\mathrm{BH}_{4}$ analogues, ADDP and HMP, cause vasorelaxation in pulmonary arteries that have been depleted of endogenous levels of $\mathrm{BH}_{4}$ by incubation with DAHP. ADDP-induced and HMP-induced relaxation was completely abolished by incubation with L-NAME indicating that relaxation is due to NO formation from 
NOS. This is consistent with the generation of NO by ADDP demonstrated in cultured cells (Suckling et al., 2008). Since ADDP and HMP did not modify vasorelaxation induced by the NO donor spermine NONOate, the analogues are not acting via potentiation of NO already present within the artery environment. Since ADDP and HMP did not cause any vasorelaxation when arteries retained their normal $\mathrm{BH}_{4}$ content, it is likely that ADDP and HMP cannot displace $\mathrm{BH}_{4}$ from NOS but may substitute for $\mathrm{BH}_{4}$ when the pterin site is unoccupied. It is likely that the two analogues of $\mathrm{BH}_{4}$ in higher oxidation states undergo an intracellular reduction reaction to form the active molecule that makes functionally competent NOS (Suckling et al., 2008).

Carbachol was still able to induce a full relaxation of pulmonary artery rings after depletion of BH4 with DAHP, and treatment with ADDP did not significantly change the carbachol concentrationresponse curve. The explanation is probably that stimulation of muscarinic receptors gives a powerful activation of eNOS that is supramaximal. Certainly NO formation exceeds the amount required for maximal relaxation in rat mesenteric artery (Simonsen et al., 1999). Thus even though eNOS is impaired a significant NO generating capacity remains. This implies that the DAHP treatment has not fully eliminated $\mathrm{BH} 4$ from the vascular wall.

$\mathrm{BH}_{4}$ produced no relaxation in pulmonary arteries even when their endogenous $\mathrm{BH}_{4}$ content had been depleted, and in fact produced significant contraction in pulmonary arteries at the higher concentrations studied. The effects of $\mathrm{BH}_{4}$ were demonstrated for the first time in the current work in isolated pulmonary arteries. In addition to the direct contractile effect of $\mathrm{BH}_{4}$, incubation with $\mathrm{BH}_{4}$ resulted in reduction in the potency of endothelium-dependent vasorelaxation with carbachol in pulmonary arteries. The contraction seen with direct administration of $\mathrm{BH}_{4}$ was consistent with what has been observed in the dog basilar artery (Kinoshita and Katusic, 1996) and in aortic rings (Yang et al., 2003). In oxygenated buffer at physiological $\mathrm{pH}, \mathrm{BH}_{4}$ undergoes autoxidation, which can generate superoxide (Blair and Pearson 1974; Kirsch et al., 2003; Nishikimi 1975). However, incubation with the cell-permeable SOD mimetic, MnTMPyP, had no effect on the contraction seen with $\mathrm{BH}_{4}$ in pulmonary arteries in our study. This indicates that the $\mathrm{BH}_{4}$-induced contraction is unlikely to involve superoxide formation.

In ADDP-treated rats there was significant potentiation of calcium ionophore A23187-induced relaxation in both normoxic and hypoxic rat lungs when compared to the respective normoxic and hypoxic rat lungs of the untreated group. This indicates that chronic treatment with ADDP resulted in improvement of NO-mediated pulmonary artery dilation. On chronic administration this improvement in pulmonary endothelial function was found not only in the pulmonary hypertensive rats but also in normotensive rats. In normotensive rats, there was enhanced expression of eNOS in the pulmonary vascular endothelium, which may explain the improved response to calcium ionophore A23187 found in vivo despite the carbachol response in vitro being unchanged. Since pulmonary hypertension raised expression of eNOS in the pulmonary vascular endothelium to a high level, it is unsurprising that ADDP treatment was unable to further increase eNOS expression in chronically hypoxic rats. In pulmonary hypertension, eNOS expression is upregulated in pulmonary arteries, although the output of NO is diminished due to uncoupling of NOS (Demiryürek et al., 2000; Weerackody et al., 2009). Thus pulmonary hypertension is able to develop despite the increased eNOS protein present in the pulmonary circulation. Nevertheless in the chronically hypoxic rat ADDP administration did improve calcium ionophore A23187induced relaxation. This may indicate improved performance of eNOS in the pulmonary vascular endothelium in pulmonary hypertension.

In conclusion, this study identifies two analogues of $\mathrm{BH}_{4}$, which can act as oxidatively stable alternatives to $\mathrm{BH}_{4}$ in causing NOmediated vasorelaxation. Treatment with ADDP improved endothelium-dependent vasorelaxation in isolated perfused lungs from both normoxic and hypoxic rats and increased eNOS expression in these rats.

\section{Acknowledgements}

PHM was supported by the British Heart Foundation (FS2001056). SK was supported by a University of Strathclyde John Anderson Scholarship and by the Overseas Research Student Support Scheme. We thank Dr Allan McPhaden, Department of Pathology, Glasgow Royal Infirmary for validation of the immunohistochemistry scoring.

\section{References}

Al Hassan, S.S., Cameron, R.J., Curran, A.W.C., Lyall, W.J.S., Nicholson, S.H., Robinson, D.R. Stuart, A., Stirling, I., Suckling, C.J., Wood, H.C.S., 1985. Specific inhibitors in vitamin biosynthesis. Part 7 Synthesis of blocked 7, 8-dihydropteridines. J. Chem. Soc. Perkin Trans. 1, 1645-1659.

Bagi, Z., Koller, A., 2003. Lack of nitric oxide mediation of flow-dependent arteriolar dilation in type I diabetes is restored by sepiapterin. J. Vasc. Res. 40, 47-57.

Blair, J.A., Pearson, A.J., 1974. Some observations on effects of light and solvent polarity on kinetics of tetrahydribiopterin autoxidation. J. Chem. Soc. Perkin Trans. 2, 1786-1787.

Bömmel, H.M., Reif, A., Fröhlich, L.G., Frey, A., Hofmann, H., Marecak, D.M., Groehn, V., Kostsonis, P., La, M.L., Koster, S., Meinecke, M., Bernhardt, M., Weeger, M., Ghisla, S. Prestwich, G.D., Pfleiderer, W., Schmidt, H.H.W., 1998. Anti-pterins as tools to characterise the function of tetrahydrobiopterin in NO synthase. J. Biol. Chem. 273, 33142-33149.

Cosentino, F., Hürlimann, D., Delli Gatti, C., Chenevard, R., Blau, N., Alp, N.J., Channon, K.M., Eto, M., Lerch, P., Enseleit, F., Ruschitzka, F., Volpe, M., Lüscher, T.F., Noll, G. 2008. Chronic treatment with tetrahydrobiopterin reverses 4 endothelial dysfunction and oxidative stress in hypercholesterolaemia. Heart 9, 487-492.

Crabtree, M.J., Tatham, A.L., Al-Wakeel, Y., Warrick, N., Hale, A.B., Cai, S., Channon, K.M., Alp, N.J., 2009. Quantitative regulation of intracellular endothelial nitric-oxide synthase (eNOS) coupling by both tetrahydrobiopterin-eNOS stoichiometry and biopterin redox status. J. Biol. Chem. 284, 1136-1144.

Demiryürek, A.T., Karamsetty, M.R., McPhaden, A.R., Wadsworth, R.M., Kane, K.A. MacLean, M.R., 2000. Accumulation of nitrotyrosine correlates with endothelial NO synthase in pulmonary resistance arteries during hypoxia in the rat. Pulmon. Pharmacol. Ther. 13, 157-165.

Demoncheaux, E.A.G., Higenbottam, T.W., Kiely, D.G., Wong, J.M., Wharton, S., Varcoe, R. Siddons, T., Spivey, A.C., Hall, K., Gize, A.P., 2005. Decreased whole body endogenous nitric oxide production in patients with primary pulmonary hypertension. J. Vasc. Res. 42, 133-136.

Eskurza, I., Myerburgh, L.A., Kahn, Z.D., Seals, D.R., 2005. Tetrahydrobiopterin augments endothelium-dependent dilatation in sedentary but not in habitually exercising older adults. J. Physiol. 568, 1057-1065.

Farrow, K.N., Lakshminrusimha, S., Reda, W.J., Wedgwood, S., Czech, L., Gugino, S.F., Davis, J.M., Russell, J.A., Steinhorn, R.H., 2008. Superoxide dismutase restores eNOS expression and function in resistance pulmonary arteries from neonatal lambs with persistent pulmonary hypertension. Am. J. Physiol. Lung Cell. Mol. Physiol. 295 L979-L987.

Fitzal, F., Redl, H., Strohmaier, W., Werner, E.R., Bahrami, S., 2002. A 4-amino analogue of tetrahydrobiopterin attenuates endotoxin-induced hemodynamic alterations and organ injury in rats. Shock 18, 158-162.

Francis, B.N., Wilkins, M.R., Zhao, L., 2009. Tetrahydrobiopterin prevents pulmonary hypertension in monocrotaline rat model. Am. J. Respir. Crit. Care Med. 179, A1821.

Fröhlich, L.G., Kotsonis, P., Traub, H., Taghavi-Moghdam, S., Al Masoudi, N., Hofmann, H., Strobel, H., Matter, H., Pfleiderer, W., Schmidt, H.H.W., 1999. Inhibition of neuronal nitric oxide synthase by 4 -aminopteridine derivatives: structure-activity relationship of antagonists of (6R)-5, 6, 7, 8-tetrahydrobiopterin cofactor. J. Med. Chem. 42, 4108-4121.

Gibraeil, H.D., Dittrich, P., Saleh, S., Mayer, B., 2000. Inhibition of endotoxin-induced vascular hyporeactivity by 4-amino-tetrahydrobiopterin. Br. J. Pharmacol. 131, 757-1765.

Gorren, A.C.F., Schrammel, A., Reithmüller, C., Schmidt, K., Koesling, D., Werner, E.R. Mayer, B., 2000. Nitric oxide-induced autoinhibition of neuronal nitric oxide synthase in the presence of the autoxidation-resistant pteridine 5-mthyltetrahydrobiopterin. Biochem. J. 347, 475-484.

Grant, S., Lutz, E.M., McPhaden, A.R., Wadsworth, R.M., 2006. Location and function of VPAC1, VPAC2 and NPR-C receptors in VIP-induced vasodilation of porcine basilar arteries. J. Cereb. Blood Flow Metab. 26, 58-67.

Grobe, A.C., Wells, S.M., Benavidez, E., Oishi, P., Azakie, A., Fineman, J.R., Black, S.M., 2006. Increased oxidative stress in lambs with increased pulmonary blood flow and pulmonary hypertension: role of NADPH oxidase and endothelial NO synthase. Am J. Physiol. Lung Cell. Mol. Physiol. 290, L1069-L1077.

Heitzer, T., Brockhoff, C., Mayer, B., Warnholtz, A., Mollnau, H., Henne, S., Meinertz, T. Munzel, T., 2000a. Tetrahydrobiopterin improves endothelium-dependent vasodilation in chronic smokers - evidence for a dysfunctional nitric oxide synthase. Circ. Res. 86, E36-E41.

Heitzer, T., Krohn, K., Albers, S., Meinertz, T., 2000b. Tetrahydrobiopterin improves endothelium-dependent vasodilation by increasing nitric oxide activity in patients with Type II diabetes mellitus. Diabetologia 43, 435-1438.

Higashi, Y., Sasaki, S., Nakagawa, K., Kimura, M., Noma, K., Hara, K., Jitsuiki, D., Goto, C. Oshima, T., Chayama, K., Yoshizumi, M., 2006. Tetrahydrobiopterin improves aging- 
related impairment of endothelium-dependent vasodilation through increase in nitric oxide production. Atherosclerosis 186, 390-395.

Hyndman, M.E., Verma, S., Rosenfeld, R.J., Anderson, T.J., Parsons, H.G., 2002. Interaction of 5-methyltetrahydrofolate and tetrahydrobiopterin on endothelial function. Am. J. Physiol. Heart Circ. Physiol. 282, H2167-H2172.

Khoo, J.P., Zhao, L., Alp, N.J., Bendall, J.K., Nicoli, T., Rockett, K., Wilkins, M.R., Channon, K.M., 2005. Pivotal role for endothelial tetrahydrobiopterin in pulmonary hypertension. Circulation 111, 2126-2133.

Kinoshita, H., Katusic, Z.S., 1996. Exogenous tetrahydrobiopterin causes endotheliumdependent contractions in isolated canine basilar artery. Am. J. Physiol. Heart Circ. Physiol. 271, H738-H743.

Kinoshita, H., Milstien, S., Wambi, C., Katusic, Z.S., 1997. Inhibition of tetrahydrobiopterin biosynthesis impairs endothelium-dependent relaxations in canine basilar artery. Am. J. Physiol. Heart Circ. Physiol. 273, H718-H724.

Kirsch, M., Korth, H.G., Stenert, V., Sustmann, R., de Groot, H., 2003. The autoxidation of tetrahydrobiopterin revisited - proof of superoxide formation from reaction of tetrahydrobiopterin with molecular oxygen. J. Biol. Chem. 278, 24481-24490.

Kotsonis, P., Fröhlich, L.G., Raman, C.S., Li, H., Berg, M., Gerwig, R., Groehn, V., Kang, Y., Al-Masoudi, N., Taghavi-Moghadam, S., Mohr, D., Münch, U., Schnabel, J., Martásek, P., Masters, B.S.S., Strobel, H., Poulos, T., Matter, H., Pfleiderer, W., Schmidt, H.H.H.W., 2001. Structural basis for pterin antagonism in nitric-oxide synthase: development of novel 4-oxo-pteridine antagonists of (5R)-5, 6, 7, 8-tetrahydrobiopterin. J. Biol. Chem. 276, 49133-49141.

Nandi, M., Leiper, J., Arrigoni, F., Hislop, A., Vallance, P., Haworth, S., 2006. Developmental regulation of GTP-CH1 in the porcine lung and its relationship to pulmonary vascular relaxation. Pediatr. Res. 59, 767-772.

Nishikimi, M., 1975. Generation of superoxide anion in reaction of the tetrahydropteridines with molecular oxygen. Arch. Biochem. Biophys. 166, 273-279.
Pannirselvam, M., Simon, V., Verma, S., Anderson, T., Triggle, C.R., 2003. Chronic oral supplementation with sepiapterin prevents endothelial dysfunction and oxidative stress in small mesenteric arteries from diabetic (db/db) mice. Br. J. Pharmacol. 140, 701-706.

Setoguchi, S., Hirooka, Y., Eshima, K., Shimokawa, H., Takeshita, A., 2002. Tetrahydrobiopterin improves impaired endothelium-dependent forearm vasodilation in patients with heart failure. J. Cardiovasc. Pharmacol. 39, 363-368.

Simonsen, U., Wadsworth, R.M., Buus, N.H., Mulvany, M.J., 1999. In vitro simultaneous measurements of relaxation and nitric oxide concentration in rat superior mesenteric artery. J. Physiol. 271-282 516.1.

Suckling, C.J., Gibson, C.L., Huggan, J.K., Morthala, R.R., Clarke, B., Kunuthur, S., Wadsworth, R.M., Daff, S., Papale, D., 2008. 6-Acetyl-7, 7-dimethyl-5, 6, 7, 8-tetrahydropterin is an activator of nitric oxide synthases. Bioorg. Med. Chem. Lett. 18, 1563-1566.

Tiefenbacher, C.P., Bleeke, T., Vahl, C., Amann, K., Vogt, A., Kubler, W., 2000. Endothelial dysfunction of coronary resistance arteries is improved by tetrahydrobiopterin in atherosclerosis. Circulation 102, 2172-2179.

Vasquez-Vivar, J., Martasek, P., Whitsett, J., Joseph, J., Kalyanaraman, B., 2002. The ratio between tetrahydrobiopterin and oxidized tetrahydrobiopterin analogues controls superoxide release from endothelial nitric oxide synthase: an EPR spin trapping study. Biochem. J. 362, 733-739.

Wang, S.X., Xu, J., Song, P., Wu, Y., Zhang, J.H., Choi, H.C., Zou, M.H., 2008. Acute inhibition of guanosine triphosphate cyclohydrolase 1 uncouples endothelial nitric oxide synthase and elevates blood pressure. Hypertension 52, 484-490.

Weerackody, R.P., Welsh, D.J., Wadsworth, R.M., Peacock, A.J., 2009. Inhibition of p38 MAP kinase reverses hypoxia induced pulmonary artery endothelial dysfunction. Am. J. Physiol. Heart Circ. Physiol. 296, H1312-H1320.

Yang, D., Levens, N., Zhang, J.N., Vanhoutte, P.M., Feletou, M., 2003. Specific potentiation of endothelium-dependent contractions in SHR by tetrahydrobiopterin. Hypertension 41, 136-142. 